\title{
Terahertz Wave Emission from Layered Superconductors: Interferometer Measurements
}

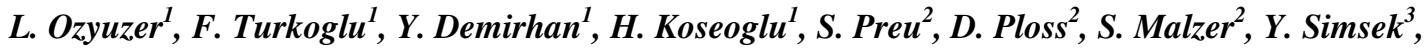 \\ H. B. Wang ${ }^{4}$, and P. Muller ${ }^{3}$
}

\author{
${ }^{1}$ Department of Physics, Izmir Institute of Technology, 35430 Urla Izmir, Turkey, ozyuzer@iyte.edu.tr \\ ${ }^{2}$ Max Planck Optics Group, University of Erlangen-Nurnberg, Erlangen, Germany \\ ${ }^{3}$ Physical Institute III, University of Erlangen-Nurnberg, Erlangen, Germany \\ ${ }^{4}$ National Institute for Materials Science, Tsukuba, Japan
}

\begin{abstract}
Rectangular $\mathrm{Bi}_{2} \mathrm{Sr}_{2} \mathrm{CaCu}_{2} \mathrm{O}_{8+\delta}$ (Bi2212) mesa structures were fabricated on as-grown Bi2212 single crystal superconductors using standard photolithography and $\mathrm{Ar}$ ion beam etching techniques. We have performed c-axis resistance versus temperature (R-T), current-voltage (I-V) characteristics and bolometer measurements. Furthermore, in contrast to previous studies, the emission frequency was determined using interferometer set up instead of FTIR. The interference patterns were detected outside the cryostat after traveling long way through ambient space. The emission frequency calculated by Fourier transform of interference data is consistent with Josephson frequencyvoltage relation.
\end{abstract}

\section{Introduction}

There is a growing interest in technology of electromagnetic waves in terahertz frequency region $(0.1-10 \mathrm{THz})$ due to their variety of application areas in the physical, astronomical, medical and biological sciences, including imaging, spectroscopy, information technology, environmental monitoring and medical diagnosis [1]. However, these applications have been limited by the scarcity of inexpensive compact solid-state sources of powerful continuous wave $\mathrm{THz}$ radiation.

The ac Josephson effect occurring between two superconducting electrodes separated by a thin insulating layer provides a unique way to develop voltage-tunable generators of electromagnetic radiation in the terahertz frequency range. When a bias voltage is applied to a Josephson junction, it behaves as a source of high-frequency electromagnetic radiation whose frequency is governed by the ac Josephson relation $\mathrm{f}_{\mathrm{Jos}}=\mathrm{V} / \Phi_{0}$, where $\mathrm{V}$ is the voltage across the junction and $\Phi_{\mathrm{o}}$ is flux quantum. Therefore, $1 \mathrm{mV}$ corresponds to $0.483 \mathrm{THz}$. However, the operation frequency is restricted by the superconducting gap, so it cannot exceed several hundred gigahertz for the devices made of conventional superconductors [2]. Also, the power emitted from a single junction is too small for practical applications, typically in the order of $\mathrm{pW}$. Large series arrays of conventional Josephson junctions could emit much larger power, if they radiate coherently [3]. However, the synchronization is necessary and different junction parameters may cause desynchronized oscillations in junctions.

Natural stacks of Josephson tunnel junctions in layered high temperature superconductors, such as $\mathrm{Bi}_{2} \mathrm{Sr}_{2} \mathrm{CaCu}_{2} \mathrm{O}_{8+\delta}$ (Bi2212), are very promising devices for developing coherent sources of THz radiation. Since the intrinsic Josephson junctions (IJJs) are homogeneous in the atomic scale along the c-axis of Bi2212 single crystals, highly-uniform junction arrays hold great potential for very high emission power, and the large value of the superconducting gap, typically of tens of meV, covers the whole terahertz frequency band. Many instructive ideas have been proposed and tried to achieve synchronized $\mathrm{THz}$ radiation from intrinsic Josephson junctions such as applying a magnetic field to induce coherent Josephson vortex flow [4], the use of shunting elements, inserting the Bi2212 crystal into a microwave cavity. However, the far-field radiation power obtained from Bi2212 is limited to the pW range.

Recently, we succeeded in detecting strong, coherent, and continuous-wave radiation in the THz frequency range emitted from the rectangular mesa structure fabricated on top of the Bi2212 surface without an applied magnetic field [5]. Since the width of mesa is smaller than the propagation distance of the Josephson plasma waves, mesa behaves like a cavity. When a resonance condition is approached by scanning the applied voltage, oscillating 
Josephson current in an individual IJJ synchronized with the help of cavity resonance and this leads to increase of the coherent in-phase oscillation of more junctions. When the Josephson frequency of the IJJ matches one of the cavity resonance modes of Josephson plasma wave inside the mesa, resonance condition occurs and almost all of the IJJ synchronized into a coherent in-phase oscillation. We observed that the fundamental frequencies of the emission were as high as $0.85 \mathrm{THz}$ and radiation power was up to $0.5 \mu \mathrm{W}$. More recently, emission powers of $5 \mu \mathrm{W}$ and frequencies at the higher harmonics up to $2.5 \mathrm{THz}$ have been obtained. These encouraging progresses inspire further investigations. Kurter et al. [6] presented thermal analysis of the mesa structure. Tunability of the THz emission and angular dependence of the emission power have also been studied [7]. Using low temperature scanning laser microscopy, Wang et al. [8] imaged electric field distributions in the junction stack of Bi2212 and observed standing electromagnetic waves (cavity resonances). Fabrication of clear rectangular mesas with sharp edges [9] and emission characteristics of $\mathrm{THz}$ emitting mesas were reported. Hot spot is another important theme and is under intense investigation [10].

\section{Experiment}

In this work, experiments were performed on as-grown Bi2212 single crystals which were grown using Traveling Solvent Floating Zone growth technique (TSFZ). Rectangular mesa structures with $55 \mu \mathrm{m}$ width and 300 $\mathrm{m}$ long were fabricated on Bi2212 single crystals using standard photolithograph and Ar ion beam etching techniques. In order to make a contact on top of the mesas, firstly an insulating layer $\left(\mathrm{CaF}_{2}\right)$ deposited onto the crystal and a small area of the mesa. After that, a gold stripe with the width of $30 \mu \mathrm{m}$ was created by lift-off technique on the mesa and $\mathrm{CaF}_{2}$ layer. Finally three gold probe wires were connected to the two contact paths and mesa by silver epoxy.

In order to characterize the Bi2212 mesas, c-axis resistance versus temperature (R-T) and current-voltage (I-V) behavior were measured in a He flow cryostat. During I-V characterization, Si composite bolometer was used to detect the emission. THz interferometer set up shown in Fig. 1 was used to find the emission frequency of the mesa. This system was controlled by Labview program. When we apply certain voltage in the emission region, this set up splits a single wave coming from long edge of mesa, then brings the constituent waves back together so that they superpose, forming an interference pattern. Here we used a Si wafer as a beam splitter.

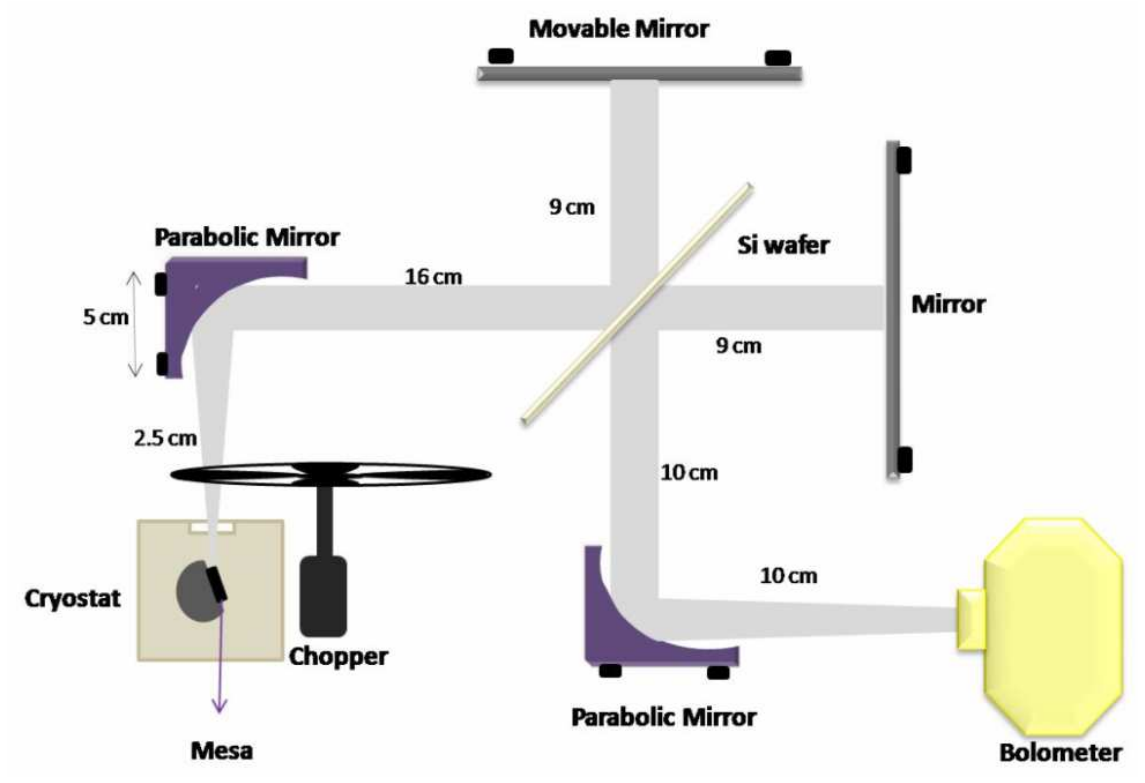

Fig. 1 Interferometer setup for frequency determination.

\section{Results: I-V and Bolometer-V Measurements}

Figure 2(a) shows a typical I-V characteristics of one of the THz emitting mesas with $55 \times 300 \mu \mathrm{m}^{2}$ at $\mathrm{T}=22 \mathrm{~K}$. The backbending of the I-V curve at high voltages is due to self heating effects as indicated by the appearance of unpolarized blackbody radiation. Figure 2(b) shows emission characteristics of mesa. When I-V curve shows backbending, the bolometer detects the heating of the mesa in the form of unpolarized blackbody radiation. When all junctions, in this case 534, are in the resistive state and the bias is decreasing slowly, the bolometer begins to give a 
response at $0.66 \mathrm{~V}$. Further decreasing the bias, the signal reaches a peak position around 0.64 V. Polarized emission peak for negative bias voltages can be also seen. On the bias decreasing part of the I-V curve at low bias there are some jumps, which occur as some junctions switch to the zero voltage state. These jumps are called as re-trapping. We did not observe re-trapping event in the emission region, this indicates that all junctions are locked to resistive state.

In addition, maximum bolometer response value of the emission peak can be seen in the unpolarized thermal radiation region at $1410 \mathrm{mV}$. If we think this thermal radiation detected by the bolometer as blackbody radiation from the mesa, we can estimate the emission power. To do so, we need to determine the local temperature of unpolarized infrared radiation at $1410 \mathrm{mV}$, which equals V/I, along the I-V curve. However, such a high resistance cannot be observed in temperature-dependent mesa resistance, so, we extrapolated the curve to lower $\mathrm{T}$ and determined the temperature of unpolarized radiation as $\mathrm{T}=71.9 \mathrm{~K}$. Modified Stefan-Boltzmann law was used to calculate expected blackbody radiation from the Bi2212 surfaces. The emitted power is given by the modified Stefan-Boltzmann law. Assuming we collect all the radiation, we get $72.6 \mathrm{nW}$ peak power. This leads to a value of $4.65 \mu \mathrm{W}$ total power when we take into account the geometrical configuration of the equipments.
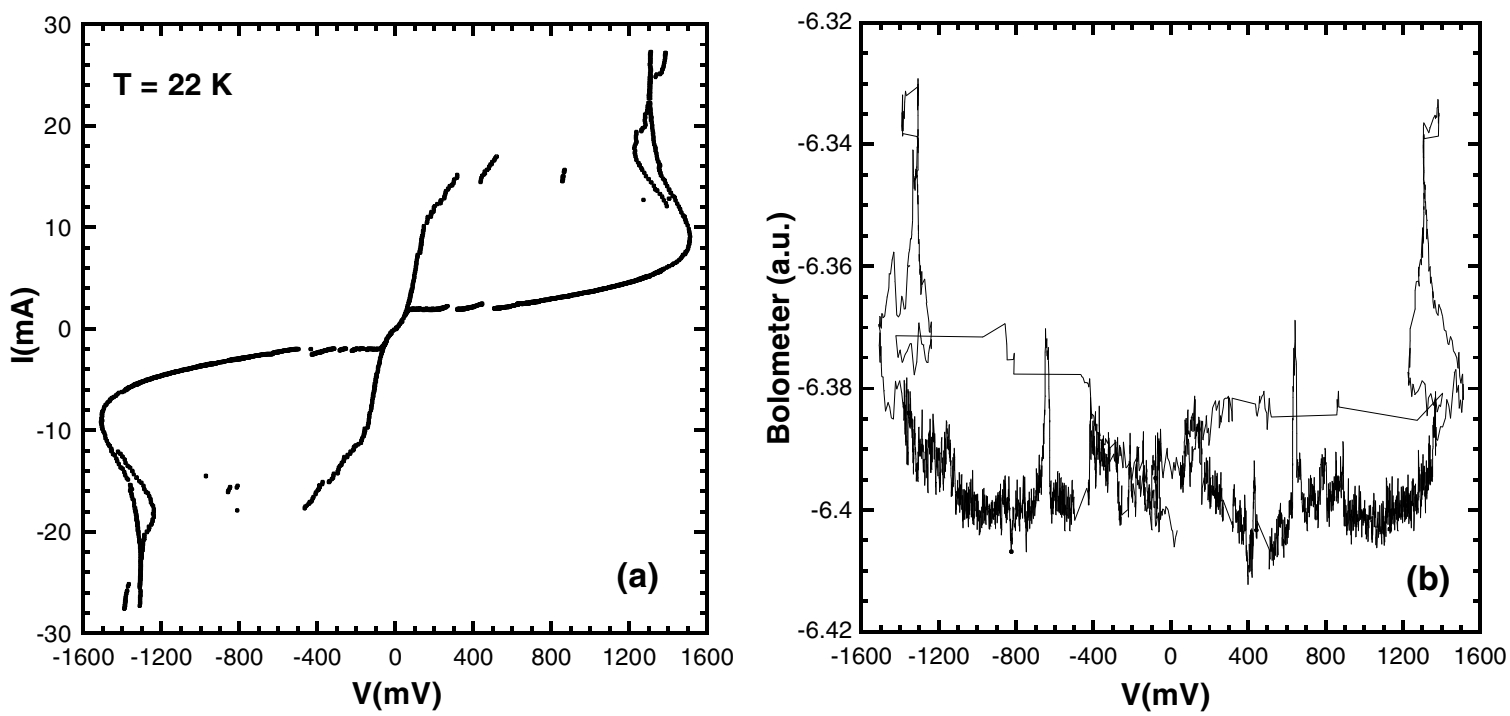

Fig. $2 \mathrm{I}-\mathrm{V}$ (a) and Bolometer-V (b) curves of the mesa at $22 \mathrm{~K}$.

\section{Results: Interferometer Measurements}

The explanation about interferometer setup was given in section 3. It is important to observe interference patterns because it simply shows coherence. THz waves coming from long edge of mesa travel $56.5 \mathrm{~cm}$ through ambient space from the mesa to the bolometer over two different paths. Since each wave passes twice between mirrors and the Si wafer, moving movable mirror a distance $\lambda / 2$ will cause a change in the phase of one wave at the bolometer. This cause passes through a minimum and return to a maximum. Figure 3(a) shows the signals detected by bolometer. When we take the fast Fourier transform (FFT) of this wave form using Labview program, we can find the emission frequency. Figure 3(b) shows the result of the frequency spectrum of the emission and the peak in this figure indicates that emission frequency is $0.537 \mathrm{THz}$. Here, the full width at half maximum (FWHM) of the peak is 31.3 $\mathrm{GHz}$. This quite large value is attributed to the instrumental origin. Moreover, there are some technical difficulties, for example, we cannot keep the voltage constant during the measurement. The width of $55 \mu \mathrm{m}$ corresponds to an emission frequency of roughly $0.530 \mathrm{THz}$ for this sample, according to the Josephson voltage-frequency relation, occurs at $586 \mathrm{mV}$ for 534 junctions. This is nearly equal to interferometer result, so it satisfies Josephson voltagefrequency relation.

\section{Conclusion}

Intrinsic Josephson junctions of layered superconductor Bi2212 was patterned as a $\mathrm{THz}$ emitting device. A simple interferometer set up was used for frequency determination. $\mathrm{THz}$ emission frequency of $55 \mathrm{~m}$ mesa was found 0.537 $\mathrm{THz}$, which is consistent with Josephson voltage-frequency relation. 

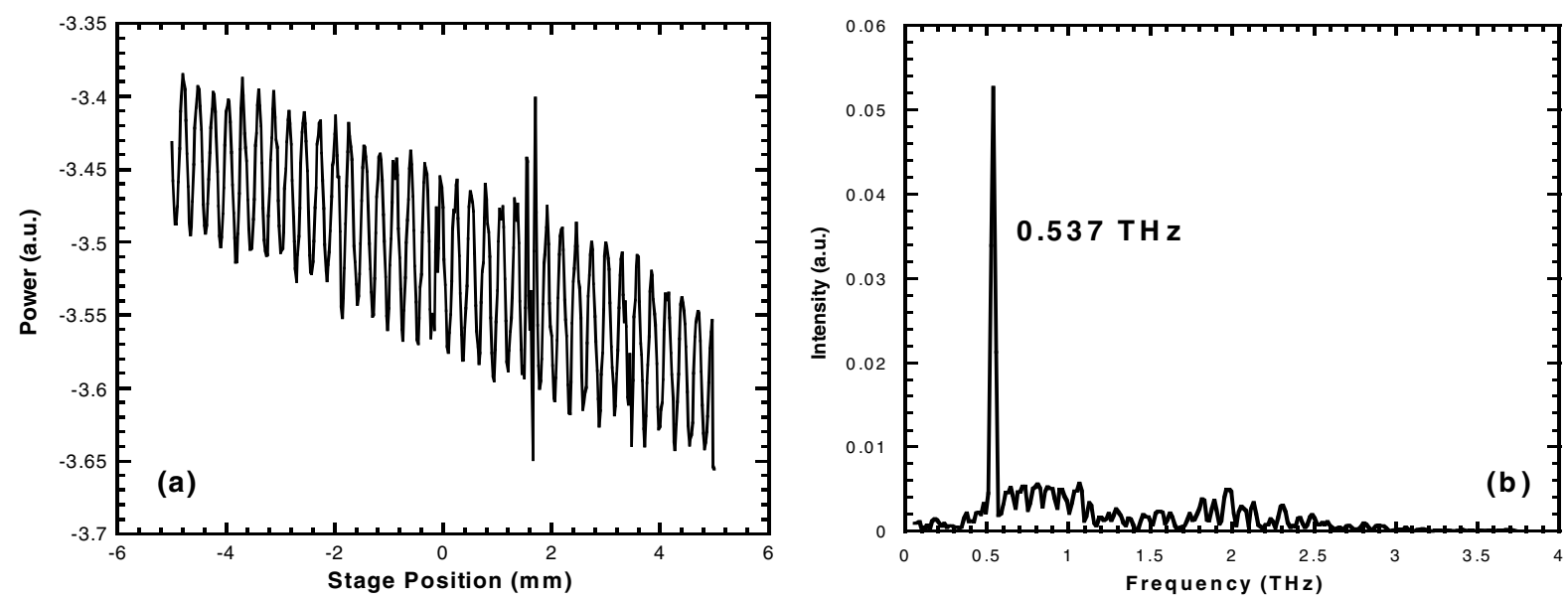

Fig. 3 Interference patterns detected by bolometer (a) and the frequency spectrum of the emission (b).

\section{Acknowledgments}

This research is supported in part by the TUBITAK (Scientific and Technical Council of Turkey) project no. 110 T248. LO acknowledges support from the Alexander von Humboldt Foundation.

\section{References}

1. M. Tonouchi “Cutting-edge terahertz technology,” Nature Photonics, 1, 2007, pp. 97.

2. L. Ozyuzer, Y. Simsek, H. Koseoglu, F. Turkoglu, C. Kurter, U. Welp, A. E. Koshelev, K. E. Gray, W. K. Kwok, T. Yamamoto, K. Kadowaki, Y. Koval, H. B. Wang, P. Müller, "Terahertz wave emission from intrinsic Josephson junctions in high-Tc superconductors," Supercond. Sci. and Technol., 22, 2009, pp. 114009.

3. P. Barbara, P., A. B. Cawthorne, S. V. Shitov and C. J. Lobb. "Stimulated Emission and Amplification in Josephson junction Arrays," Phys. Rev. Lett., 82, 1999, pp. 1963.

4. M. Bae, H. J. Lee, J. H. Choi, "Josephson-Vortex-Flow Terahertz Emission in Layered High-T ${ }_{\mathrm{c}}$ Superconducting Single Crystals," Phys. Rev. Lett., 98, 2007, pp. 027002.

5. L. Ozyuzer, A. E. Koshelev, C. Kurter, N. Gopalsami, Q. Li, M. Tachiki, K. Kadowaki, T. Tamamoto, H. Minami, H. Yamaguchi, T. Tachiki, K. E. Gray, W. K. Kwok and U. Welp, "Emission of coherent THz radiation from superconductors," Science, 318, 2007, pp. 1291.

6. C. Kurter, K. E. Gray, J. F. Zasadzinski, L. Ozyuzer, A. E. Koshelev, Q. Li, T. Yamamoto, K. Kadowaki, W. K. Kwok, M. Tachiki and U. Welp "Thermal Management in Large Bi2212 Mesas Used for Terahertz Sources," IEEE Transactions on Applied Superconductivity, 19, 2009, pp. 428.

7. K. Kadowaki, M. Tsujimoto, K. Yamaki, T. Yamamoto, T. Kashiwagi, H. Minami, M. Tachiki, and R. A. Klemm, "Evidence for a Dual-Source Mechanism of Terahertz Radiation from Rectangular Mesas of Single Crystalline $\mathrm{Bi}_{2} \mathrm{Sr}_{2} \mathrm{CaCu}_{2} \mathrm{O} 8+\delta$; Intrinsic Josephson Junctions," J. Phys. Soc. Jpn., 79, 2010, pp. 023703.

8. H. B. Wang, S. Guenon, J. Yuan, A. Iishi, S. Arisawa, T. Hatano, T. Yamashita, D. Koelle and R. Kleiner. "Hot Spots and Waves in $\mathrm{Bi}_{2} \mathrm{Sr}_{2} \mathrm{CaCu}_{2} \mathrm{O}_{8}$ Intrinsic Josephson Junction Stacks: A Study by Low Temperature Scanning Laser Microscopy," Physical Review Letters, 102, 2009, pp. 017006.

9. H. Koseoglu, F. Turkoglu, Y. Simsek, L. Ozyuzer, “The Fabrication of THz Emitting Mesas by Reactive Ion-beam Etching of Superconducting Bi2212 with Multilayer Masks," Journal of Superconductivity and Novel Magnetism, DOI 10.1007/s10948-010-0859-9.

10. H. B. Wang, S. Guénon, B. Gross, J. Yuan, Z. G. Jiang, Y. Y. Zhong, M. Grünzweig, A. Iishi, P. H. Wu, T. Hatano, D. Koelle, and R. Kleiner "Coherent Terahertz Emission of Intrinsic Josephson Junction Stacks in the Hot Spot Regime," Phys. Rev. Lett., 105, 2010, pp. 057002. 